\title{
Alternative anesthetic management of video-assisted thoracic surgery for spontaneous breathing
}

\author{
Jong Hoon Yeom and Seongho Park \\ Department of Anesthesiology and Pain Medicine, Guri Hospital, Hanyang University College of Medicine, Guri, \\ Korea
}

One-lung ventilation via double-lumen tube or a bronchial blocker with general anesthesia is known to be the golden standard in thoracic surgery. However, conventional methods of general anesthesia using muscle relaxants may induce ventilator-associated lung injury and intubation-associated complications.

Recently, other anesthetic methods have been applied and associated studies are actively being conducted. Non-intubated thoracic surgery with local or regional analgesia or monitored anesthetic care with self-breathing has been studied to avoid the complications associated with conventional general anesthesia. These anesthetic methods have been successfully employed in patients with pneumothorax, emphysema, and even oncologic conditions like peripheral lung nodules and mediastinal tumors [1-3].

The authors of the Korean Journal of Anesthesiology demonstrated thoracic epidural anesthesia with a target-controlled infusion of remifentanil and propofol as a feasible strategy for sedation in thoracoscopic surgery for decortication of empyema [4].

However, regional anesthetic methods such as thoracic epidural anesthesia, paravertebral block, or intercostal block have certain limitations and could induce some complications [5]. The choice of these alternatives might not always be available, especially in cases with bleeding tendency, poor cardiac function, or complicated respiratory function.

The selection of awake state, supraglottic airway, single-lu- men tube, bronchial blocker, or double-lumen tube for airway management should be made considering the potential problems including patient condition, operative procedure, and operated and non-operated lung conditions before the surgery. Awake state and supraglottic airway could avoid intubation-associated complications, but there might be difficulties in the removal of tracheal secretions and airway management. The double-lumen tube is stiffer, has a larger outer diameter, and may be more intolerable compared with the single-lumen tube. However, the double-lumen tube may be preferable over the single-lumen tube for specific conditions.

The anesthesiologist should choose an anesthetic method that minimizes the risks to the patient before surgery and be familiar with the variety of procedures to be performed. If spontaneous breathing is needed and regional anesthetic techniques cannot be applied, anesthetic management using the combination of remifentanil and midazolam with ketamine for spontaneous breathing may be a good alternative.

\section{Conflicts of Interest}

No potential conflict of interest relevant to this article was reported.

Corresponding author: Jong Hoon Yeom, M.D., Ph.D.

Department of Anesthesiology and Pain Medicine, Guri Hospital, Hanyang University College of Medicine, 153 Gyeongchun-ro, Guri 11923, Korea Tel: 82-31-560-2400, Fax: 82-31-563-1731, Email: yeomjh@hanyang.ac.kr

ORCID: https://orcid.org/0000-0003-3159-2072

Received: November 5, 2018. Accepted: November 13, 2018.

Korean J Anesthesiol 2019 April 72(2): 196-197

https://doi.org/10.4097/kja.d.18.00312

(c) This is an open-access article distributed under the terms of the Creative Commons Attribution Non-Commercial License (http://creativecommons.org/ licenses/by-nc/4.0/), which permits unrestricted non-commercial use, distribution, and reproduction in any medium, provided the original work is properly cited. 


\section{Author Contributions}

Jong Hoon Yeom (Supervision; Writing - original draft)

Seongho Park (Writing - review \& editing)"

\section{ORCID}

Jong Hoon Yeom, https://orcid.org/0000-0003-3159-2072

Seongho Park, https://orcid.org/0000-0003-3664-2251

\section{References}

1. Kiss G, Castillo M. Nonintubated anesthesia in thoracic surgery: general issues. Ann Transl Med 2015; 3: 110.

2. Liu J, Cui F, Li S, Chen H, Shao W, Liang L, et al. Nonintubated video-assisted thoracoscopic surgery under epidural anesthesia compared with conventional anesthetic option: a randomized control study. Surg Innov 2015; 22: 123-30.

3. Iwata Y, Hamai Y, Koyama T. Anesthetic management of nonintubated video-assisted thoracoscopic surgery using epidural anesthesia and dexmedetomidine in three patients with severe respiratory dysfunction. J Anesth 2016; 30: 324-7.

4. Moon EJ, Go YJ, Chung JY, Yi JW. Non-intubated thoracoscopic surgery for decortication of empyema under thoracic epidural anesthesia: a case report. Korean J Anesthesiol 2017; 70: 341-4.

5. Park SY. Non-intubated thoracic surgery under thoracic epidural anesthesia. Korean J Anesthesiol 2017; 70: 235-6. 